\title{
Water's Non-Tetrahedral Side
}

\author{
Richard H. Henchman ${ }^{* a b c}$ and Stuart J. Cockram ${ }^{b}$
}

Received Xth XXXXXXXXXX 20XX, Accepted Xth XXXXXXXXX 20XX

First published on the web $X$ th $X X X X X X X X X X$ 20XX

DOI: $10.1039 / \operatorname{co00000x}$

The case for liquid water having non-tetrahedral as well as tetrahedral coordination is put forward. Given the dependence of structure on the hydrogen bond definition, a recent conceptual breakthrough has been the topological hydrogen bond definition which overcomes the shortcomings of traditional cut-off-based hydrogen bond definitions. It identifies the hydrogen bonds in water's first coordination shell using assumed transition states as boundaries instead of fixed cut-offs. Here, the topological definition is applied to liquid water to characterise the distances, angles and energies of the hydrogen bonds for the different types of coordinations found. These coordinations include bent, trigonal, tetrahedral, trigonal bipyramidal, and octahedral structures, as well as bifurcated hydrogens, bifurcated oxygens and cyclic dimers, and larger polygons. All species are shown to have properties consistent with their classification, justifying their assignments, and supporting the structure of water as a continuous, single phase mixture. However, a detailed analysis to assess the existence of the assumed transition states reveals the remarkable finding that HB switching via a bifurcated hydrogen is largely a barrierless process. The likelihood of a switch depends on both the acceptor numbers and on the proximity of a donor to its acceptor. Specifically, a donor switches uphill to an acceptor of the same or higher

\footnotetext{
0 a Manchester Interdisciplinary Biocentre, The University of Manchester, 131 Princess Street, Manchester, M1 7DN, United Kingdom. Fax: 44306 5201; Tel: 44306 5194; E-mail: henchman@manchester.ac.uk

$0 b$ School of Chemistry, The University of Manchester, Oxford Road, Manchester, M13 9PL, United Kingdom.

0c Heidelberg Institute for Theoretical Studies, Schloss-Wolfsbrunnenweg 35, 69118, Heidelberg, Germany.
} 
coordination to the starting acceptor, downhill to an acceptor of lower coordination by two or more, or sits bifurcated between two acceptors if the new acceptor has a coordination lower by only one. Which it is depends intimately on the donor molecule's oscillations and on other HB switches that control the nearby acceptors' coordinations. Finally, a search is conducted for long-range structure in water in terms of asymmetry in the distribution of the donor-acceptor bias but none is found. 


\section{Introduction}

There is still considerable disagreement about the nature of short-range structure in water, let alone that of longer-range structure. The prevailing, textbook view is that water's first coordination shell is tetrahedral, with molecules engaged in four hydrogen bonds (HBs), two of them donors and two of them acceptors. This is chiefly supported by the radial distribution functions from x-ray and neutron diffraction which show that oxygen atoms are surrounded on average by two hydrogens and four to five oxygens. ${ }^{1-3}$ This structure is also commonly rationalised by the two lone pairs on oxygen accepting one donor each. To account for the greater disorder in liquid water than in ice, those who propose tetrahedral structure add the descriptors of "distorted", "random" or "on average". ${ }^{4-9}$ However, in doing so, they implicitly admit the existence of some degree of non-tetrahedral water: "distorted" is imprecise and the four nearest neighbours, not necessarily equating to the first hydration shell, are always distorted tetrahedral to some extent; "random" omits that non-tetrahedral coordinations are unavoidable at the interfaces between disordered tetrahedra; "on average" neglects the possibility of opposing fluctuations that may be undetectable by the method of analysis, and nor does such cancellation always occur, as in the case of water's oxygen oxygen (OO) coordination number exceeding four, and in the more detailed 3D oxygen-hydrogen $(\mathrm{OH})$ and $\mathrm{OO}$ distributions first presented by Kusalik and Svishchev. ${ }^{10,11}$ They reveal a broad band of oxygens and hydrogens around the central oxygen rather than two distinct peaks as would be expected for tetrahedral structure.

Proponents of tetrahedral structure maintain that because water is a single continuous phase rather than a mixture, then only one coordination is possible and only tetrahedral coordination fits the data. ${ }^{7-9,12}$ However, this neglects the possibility of a continuous single phase consisting of multiple coordinations mixed at the molecular scale. One only has to look at the case of two miscible liquids: they are considered to constitute a single continuous phase and yet 
neutron diffraction has demonstrated that even these do not mix completely at the molecular scale. ${ }^{13}$ Indeed, evidence for patches of non-tetrahedral structure in water has been found. Many theories dating back to Röntgen ${ }^{14}$ and earlier find that mixture models better explain the maxima or minima in many of water's properties. ${ }^{15-17}$ Molecular-scale inhomogeneities in water have long been detected in numerous simulations going back to the work of Stanley and Teixeira, ${ }^{18}$ and more recently using x-ray scattering. ${ }^{19}$

Proponents of tetrahedral structure argue that non-tetrahedral fluctuations at short length and time scales are not significant because water molecules are rapidly exchanging their HBs. ${ }^{7-9,12}$ Any non-tetrahedral structure would be short-lived, such that molecules are tetrahedral on average. Moreover, such fluctuations are an artifact of the myriad of cut-offs commonly used to identify HBs. ${ }^{20}$ These arguments may be challenged on numerous grounds. Firstly, a state's stability depends on the ratio of dynamic quantities rather than directly on the dynamic quantities themselves. Secondly, the distribution of all possible coordinations gives the more complete picture of structure than the average. Thirdly, HB cut-offs may well bring about artificial short-lived non-tetrahedral structure but this does not imply that all non-tetrahedral structure is a consequence of this problem. Fourthly, non-tetrahedral water has been shown to be the very means to facilitate water's rapid dynamics; ${ }^{21-31}$ if only tetrahedral water were present, then multiple molecules would have to interconvert simultaneously in order to preserve tetrahedrality, ${ }^{16,32,33}$ an unlikely event. Fifthly, neutron diffraction shows that the structure of supercritical water is better described as trigonal rather than tetrahedral before breaking down entirely at higher pressure, ${ }^{34,35}$ so it is unlikely that trigonal structure entirely disappears at more ambient conditions. How solutes perturb water's structure complicates the debate even more. Concerning the entropy loss of hydrophobic hydration, if small, hydrophobic solutes make water more tetrahedral, as much evidence suggests, ${ }^{36-41}$ then conversely pure water must be more non-tetrahedral. Furthermore, the hydration of molecules with an uneven number of 
donors and acceptors requires non-tetrahedral water to balance up the number of donors and acceptors, as is seen in x-ray crystal structures of hydrated solutes ${ }^{42,43}$ and molecular dynamics simulations. ${ }^{44}$ Finally, for ion solvation the much debated terms of "structure-making" and "structure-breaking" rest on even weaker foundations while there is uncertainty about water itself. $^{45,46}$

Despite the conflicting evidence, the tetrahedral view of water still holds sway. One explanation for this is the lack of a consensus on a non-tetrahedral component. While many candidates have been put forward, including various clathrates and crystalline forms, which have largely been dismissed, ${ }^{15,17}$ the recurring contender has been the broken $\mathrm{HB},{ }^{18,32,47}$ also referred to as a dangling or free hydrogen. However, its cause has not been helped by a wide variety of suggested values and justifications, ${ }^{15,48}$ usually involving a cut-off in some property. The most recent well-known example, as derived from x-ray absorption, emission and scattering studies, estimated that more than half the HBs are broken. ${ }^{19,49}$ This was immediately opposed because it was inconsistent with numerous data such as the radial distribution functions described earlier, ${ }^{1-3}$ water's single-phase structure inferred from the temperature dependence of Raman spectra $^{12}$ and the transient life-times of broken HBs from femtosecond 2D IR spectroscopy. ${ }^{20,50}$ Recent electronic structure calculations indicated that x-ray absorption spectra are only sensitive to the strongest one or two HBs in the first hydration shell which invariably change rapidly due to molecular oscillation. ${ }^{51}$ Thus the spectra do not conflict with tetrahedral structure, but neither do they exclusively confirm it. A less commonly considered non-tetrahedral alternative is a water molecule with more than four HBs. ${ }^{21-25,27-31}$ The lower popularity of this structure probably reflects the common expectation that water has a maximum of four HBs, while broken HBs should be more common if HBs are frequently breaking. Giguère proposed that the a bifurcated hydrogen $(\mathrm{BH})$, or in other words, a triple donor, was the main non-tetrahedral state in water, backed up by the temperature dependence of Raman and neutron-diffraction spec- 
tra. ${ }^{21,22}$ Sciortino et al., however, studying the OO coordination, proposed that the extra fifth neighbour of a water molecule catalysed HB switching. ${ }^{23-25}$ They discussed how this species could manifest itself as a triple donor or triple acceptor but their data, ignoring hydrogens, did not make this explicit. Significant evidence that the $\mathrm{BH}$ was a transition state was found by a number of groups. ${ }^{11,20,28,29,50}$ Later work showed that the triple acceptor was much more prevalent than the $\mathrm{BH} \cdot{ }^{30,31,52}$ Nonetheless, the idea that water can have more than four HBs is still not widely considered.

To resolve the riddle of water's structure in the first hydration shell, the following questions must be answered: where are the HBs, what are broken HBs, can molecules have more than four HBs, where is the boundary between the first and second hydration shells, and what are the transition states of HB switching? We next explain how the use of cut-offs to define HBs is responsible for much of this uncertainty and demonstrate that a new approach to determine HBs based on the topology of the potential energy surface is able to answer these questions consistently.

\subsection{Traditional Hydrogen Bond Definitions and Water Structure}

The standard way to identify the HBs in the first hydration shell uses cut-offs in one or more quantities that relate to the strength of the donor-acceptor interaction. The most direct quantities are coordinate distributions in some coordinate(s) from either x-ray diffraction, ${ }^{1}$ neutron diffraction $^{3}$ or computer simulation ${ }^{49,53-57}$ or the energy distribution from computer simulation. ${ }^{18,58-60}$ Beyond these cut-offs, HBs are usually regarded as being broken. Cut-offs have their origins in the analysis of structures in which the HBs are stable, regular, and consequently sharply resolved, such as in gas-phase clusters, minimised geometries or crystal structures. For liquids, on the other hand, in which the incessant dynamics and disorder means that HBs are 
continuously distributed, it is not obvious where to place the cut-off. The resulting HB distribution is highly sensitive to the choice of cut-off, leading to unavoidable ambiguity in water's structure as has already been described. The most robust types of cut-off have a reasonable justification, such as being at the first minimum in the radial distribution function ${ }^{1,3,35,55-57}$ or at a point invariant to temperature. ${ }^{58}$ However, many other definitions are more elaborate and arbitrary. There are a range of other techniques to determine first hydration shell structure indirectly from other data such as IR, Raman ${ }^{7,12}$ or X-ray spectra ${ }^{49}$ but these make more assumptions and we will not consider them further.

Regardless of how cut-offs are chosen, their main weakness is that they cannot accurately separate the overlap between the first and second-shell distributions that invariably occurs in a liquid. If the cut-off is too small, then oscillating molecules in the first hydration shell sometimes exceed the cut-off, leading to broken HBs and single acceptors. Conversely, if the cut-off is too large, then more distant, second-shell molecules may stray temporarily within the cut-off, leading to BHs and triple acceptors. These two effects artificially broaden the HB distribution, masking any real variation that may be present in the first hydration shell. For these reasons, it has often been said that instantaneous HB determination is impossible and that the structure depends on the time scale considered. ${ }^{17,20,50,51,57}$ The cut-off problem is exacerbated when it comes to water dynamics because additional measures are usually required to determine if a HB switch has taken place, such as tighter HB criteria, averaging over a set time, or considering the time for the HB to break for the last time. ${ }^{28,29,56,61-63}$ In any case, most HB definitions that are commonly used err on the conservative side and yield about $10 \%$ broken HBs at ambient conditions, with few molecules having more than four HB. This value is in accord with the greater disorder expected in the liquid than in ice and was rationalised by Pauling in terms of the ratio of the enthalpy of sublimation to that of ice. ${ }^{64}$ However, given that water's OO coordination number is larger than that of ice, ${ }^{1-3}$ very few broken HBs might also 
be expected. In fact, femtosecond 2D IR spectroscopy suggests that HB switches are very rapid, such that there should be a negligible number of broken HBs or BHs in water. ${ }^{20,50}$ Moreover, in a remarkably under-reported inconsistency, ${ }^{44} 10 \%$ of broken HBs is surprisingly comparable to the $10-12 \%$ broken HBs at the air-water interface (equivalent to $20-25 \%$ of molecules having a broken HB) measured by vibrational sum-frequency generation, ${ }^{65}$ a system which should surely have many more broken HBs than the bulk.

All this points towards conceptual flaws in the nature of a broken HB as defined by a cutoff. Desiraju has expressed the view that the cut-off criterion "has caused more havoc to the field of hydrogen bonding than any other factor". ${ }^{6}$ HBs, being predominantly electrostatic interactions, do not suddenly drop to zero at a cut-off, as "broken" or even the word "bond" implies. Rather, they extend out continuously and diminishingly to infinity. It is understandable that most donor-acceptor interactions lying outside a reasonably chosen cut-off should not be considered as HBs because they are weaker and most such donors already have a HB to a closer acceptor. However, it seems overly restrictive that a donor-acceptor interaction lying just outside the cut-off for a donor without any acceptor is said to have a broken HB. Likewise, it would appear not restrictive enough if a donor-acceptor interaction lies just inside the cut-off when the donor already has a closer acceptor, bringing about a BH or over-coordinated oxygen. Surely what has a greater effect on whether a donor-acceptor interaction might qualify as a HB is not some fixed cut-off but rather a consideration of the interactions with neighbouring molecules, an issue we take up later.

A single test can resolve both the reality of the broken HB as well as the extent to which the HB distribution is artificially broadened by the fixed cut-off, discussed earlier. A simulation of water at ambient conditions may be quenched to remove the confounding thermal vibration but largely preserve the local HBs and structure. ${ }^{67}$ The results are that the spread of the HB 
distribution is reduced but not removed, and the broken HBs almost entirely vanish. ${ }^{20,31}$ Both results happen for the same reason because broken HBs are the main culprit for the additional broadening. This implies that a broken HB as defined by a cut-off is merely a distortion and not a distinctly different entity, failing the requirement set forth by Eisenberg and Kauzmann for a broken HB. ${ }^{15}$ Even more significantly, that the distribution does not collapse to a purely tetrahedral coordination reveals that non-tetrahedral structure is present in liquid water. This discrepancy between the quenched and ambient structures convincingly exposes the flaws of traditional HB definitions in understanding water structure. As we now explain, the best way to remove the dependence on the fixed cut-off is to remove it.

\subsection{Topological Hydrogen Bond Definition and Water Structure}

The recently proposed topological HB definition is able to address many of the limitations of traditional HB definitions described above and provides a more robust determination of the structure of water's first coordination shell. ${ }^{31}$ The definition is simple and intuitive: an electropositive hydrogen donates to the most attractive non-covalently bonded acceptor, unless there is a more attractive donor-acceptor interaction between the molecules involved, in which case the donor forms a broken HB. No cut-offs are specified in the definition which distinguishes it from its two fore-runners that assume a HOO angle of less than $30^{\circ 12}$ or a maximum of two acceptors. ${ }^{68}$ These two HB definitions are also based on the nearest-acceptor-to-adonor rather than the more general most-attractive-force. The topological definition resembles nearest-neighbour approaches which are commonly used in analysing variable structures in the condensed phase or in chemical reactions. It is applicable to any set of force field parameters and any combination of donors and acceptors, although it may be advisable to remove the repulsive Lennard-Jones term from hydrogens that have them in order to avoid short-range

repulsion. It could be used in an ab initio simulation but would require a method to define the 
charge density of an atom such as Atoms in Molecules ${ }^{69}$ or only involve distances instead of forces. ${ }^{70}$ It is generally consistent with the IUPAC HB definition ${ }^{71}$ and addresses its open-ended requirement of bond formation by assuming that a donor forms a stable interaction with its nearest acceptor.

The feature of the topological definition that explains its name is that it places the cut-off of a $\mathrm{HB}$ at the point where the donors are equidistant from two acceptors and that this $\mathrm{BH}$ structure is presumed to lie close to the transition state surface separating the two different HB states. ${ }^{20,23-25,28,29,50}$ Transition state surfaces are well-defined topological features of the potential energy surface. When a donor hydrogen is pulled equally by two acceptors, these opposing forces sum to zero in the direction of the switch, bringing about an estimate of a point lying on the full $6 N$-1-dimensional transition state surface, where $N$ is the number of water molecules. This approximation is useful because it would otherwise be intractable to locate the full, exact transition state surface together with its saddle point for a single HB switch let alone for all of them. A molecular dynamics trajectory with its discrete time-step is already approximate, and it is only necessary to know when the surface has been crossed. Other longer-range interactions contribute but these are weaker and their perturbations, being positive or negative, would cancel to a large degree. Determining HBs based on transition state surfaces is particularly advantageous in studying HB kinetics. ${ }^{31}$ Artificial recrossings are reduced without including additional procedures to ensure whether a switch has occurred as is necessary when HB cut-offs are used. ${ }^{28,29,56,61-63}$ Traditional HB definitions fare worse because they effectively assume that transition state surfaces, marked by a cut-off, are the same for every single HB switch. Because this assumption is clearly false in a liquid, this brings into being the enigmatic, cut-off-dependent broken HBs and over-coordinated water molecules. Not taking this variability into account has arguably been one of the main obstacles preventing a clear view of the structure of water. For conciseness, consistency with usage in the field, and 
because ensembles are more relevant to this analysis than specific stationary points, we will refer to transition state surfaces as transition states, in the same way that stable states are often used to refer to the ensemble rather than to only the local minimum.

By the topological definition, a donor hydrogen forms a HB to an oxygen acceptor depending on the strengths of three other types of interaction: the donor molecule's other hydrogen donating to this acceptor, either of the two hydrogens of the donor's acceptor donating back to the donor molecule, and all other acceptors. In turn, there are assumed to be three matching types of transition state which depend on whether the new acceptor is the switching water's initial acceptor, donor or neither. These are the bifurcated oxygen (BO), cyclic dimer (CD), and $\mathrm{BH}$ already mentioned. ${ }^{31}$ The $\mathrm{BO}$ has the two donors of the same water pointing to a single acceptor while the CD has a donor from each water both trying to donate to the other molecule. The BO and CD have been observed before in gas-phase dimers ${ }^{72-74}$ and larger clusters ${ }^{75}$ and found to be transition states in those cases. They have also shown up in proposed mechanisms of water ${ }^{15,62}$ and ice dynamics, ${ }^{76,77}$ in simulations of water in carbon nanotubes, ${ }^{78}$ and in electronic structure calculations in which they contribute to the enhanced pre-edge of the x-ray absorption spectrum. ${ }^{51}$ Most works have not considered them because the strained structures are outside the cut-offs of traditional HB definitions or because studies of HB networks usually assume only one HB between water molecule pairs. While BHs and symmetric BOs and CDs exist instantaneously, BOs and CDs also have asymmetric states with non-zero lifetimes comparable to half a water's libration. ${ }^{20,50,79}$ The weaker donor-acceptor interaction in these structures can be defined as broken. ${ }^{31}$ Even though the interaction strength of the broken HB is not zero, if the broken HB tries to strengthen, then the other HB has to weaken and the total energy rises. This inability for a donor to point towards its preferred acceptor, owing to a local deficiency of acceptors, meets the criterion laid out by Eisenberg and Kauzmann that a broken HB must be distinctly different to that of a HB. ${ }^{15}$ The fraction of broken HBs under ambient conditions 
is found to be only $\sim 1 \%$ which is consistent with the transient breakage time measured by experiment. ${ }^{20,50}$ At the air-water interface $\sim 20 \%$ of water molecules are predicted to have a broken HB ${ }^{44}$ again consistent with the $20-25 \%$ detectable from experiment. ${ }^{65}$ Despite these successes, there is one complication. The logic to define broken HBs for dimers implies that the strained HBs in larger polygons such as trimers and tetramers found in water ${ }^{80-82}$ could also be regarded as broken. Beyond water, multiple, strong HBs can readily exist between various pairs of molecules such as DNA bases or acetic acid dimers. This demonstrates that there is a whole spectrum of reasons to decide if a HB is broken. The case of the polygons we consider later.

Concerning the acceptor HB distribution, the topological definition predicts that about $70 \%$ of water molecules at ambient conditions have the tetrahedral coordination of two donors and two acceptors ${ }^{31}$ otherwise referred to as a double acceptor (DA). The main non-tetrahedral species are the single (SA) and triple acceptors (TA), which, because there are usually two donors, may be identified as having trigonal and trigonal bipyramidal coordinations. Trace amounts of non-acceptors (NA) and quadruple acceptors (QA) are also detected, with bent and octahedral coordinations. All such species are not new, having been seen in numerous computer simulations ${ }^{18,23-26,30,49,53-60,62}$ and x-ray crystal structures of hydrated solutes, ${ }^{83,84}$ but the difference here is that these SA and TA structures are substantial and almost equal to each other. The small difference between them is almost entirely due to the small fraction of broken HBs. Such species in many ways are consistent with the "tetrahedral on average" description of water because many of the trigonal and trigonal bipyramidal properties are opposing, making their combined effect harder to distinguish from tetrahedral structure by most forms of analysis, a prime example being the ordering of water by small hydrophobic molecules. ${ }^{36-38,40,41,44}$ However, unlike the purely tetrahedral model, the combined effect of all coordinations can better explain other properties. These include the well-known broad band of oxygen or hy- 
drogen density around the central oxygen in $3 \mathrm{D}$ probability distributions ${ }^{10,11}$ because of the planar contribution, ${ }^{31}$ and why water's OO coordination number lies between four and five. ${ }^{1-3}$ This latter effect arises because of the five-fold trigonal bipyramidal coordination combined with the greater ability of non-HB oxygens, the so-called interstitials, to approach within the coordination cut-off at the the exposed upper and lower faces of the trigonal coordination. ${ }^{11,31}$

Contributing to the strength of the assignment of HBs, the topological HB distributions are highly resistant to quenching, unlike the distributions described earlier using tradition HB definitions. ${ }^{31}$ Trigonal and trigonal bipyramidal coordinations have respectively higher or lower energy than the tetrahedral coordination, correlating with the number of HBs, while the HBs themselves are correspondingly stronger or weaker because more HBs induce more strain. The combined energy of the trigonal and trigonal bipyramidal coordinations is slightly more positive than that of the tetrahedral coordination but significantly less than the broken HB states described earlier. ${ }^{31}$ Their lifetimes are only about a third that of the tetrahedral coordination but much longer than those of the BOs and CDs. The non-tetrahedral coordinations provide a substantial number of defects that permit many more types of single-step HB switches ${ }^{27,30,31,52}$ than those that involve a donor jumping from an over to an under-coordinated acceptor. ${ }^{28,29}$ Finally, it should be noted that water's oxygen is not limited to accepting two donors because it has two lone pairs, as is commonly supposed. Based on Bernal and Fowler's early model for water $^{4}$ as well as observations of x-ray crystal structures,${ }^{85}$ electronic structure calculations ${ }^{86,87}$ and comparisons of three and four-point water models, ${ }^{8}$ Finney has emphasised that the lone pairs are largely delocalised, ${ }^{89}$ thus permitting the trigonal geometry. The same arguments also allow plenty of space on the oxygen to accept more than two HBs, particularly in water's unusually low $\mathrm{OO}$ coordination environment, thus permitting the trigonal bipyramidal geometry. Additional, well-recognised evidence for a $\mathrm{HB}$ is that it is a pre-cursor state to full proton transfer. ${ }^{71}$ Indeed, the single and triple acceptors are respective pre-cursors of the Eigen forms 
of the hydronium and hydroxide ions which are known to be three ${ }^{90}$ and five-coordinated. ${ }^{91}$

One of the main criticisms which has been levelled at the topological definition is that it is too generous in assigning HBs. In particular, it is possible for two isolated water molecules to form a $\mathrm{HB}$ at any distance or angle. This stretches the common view of HBs as being short-ranged and directional, as made clear by the many cut-off definitions described earlier. The IUPAC HB definition is less proscriptive and concerning stability only requires that there be "evidence of bond formation". ${ }^{66,71}$ This is not merely to be compatible with the term "bond", but it helps make the HB detectable and brings about structure in the first hydration shell. Such evidence qualitatively relates to HB strength such as distance, directionality, polarity, infrared stretch modulation of protein deshielding. The one exception is the suggestion that the Gibbs energy of HB formation be greater than the thermal energy. This is a disguised cut-off in that the equilibrium constant for HB formation should exceed base e, although it is softened by the absence of the specification of standard states of the donor and acceptor prior to HB formation. Arunan has advocated that the potential barrier should exceed the zero-point energy ${ }^{92}$ while other sources with a focus on weak HBs advise having no lower limit on HB strength. ${ }^{42,84,93}$ In any case, the IUPAC HB definition does not stipulate complete adherence to every criteria but rather that the more criteria that are met, the more reliable the $\mathrm{HB}$ characterisation. A second, less commonly made criticism against the topological definition, and rather more a source of discomfort to its proposers, is that it has a disguised cut-off in allowing no more than one acceptor per donor. In other words, the possibility of a $\mathrm{BH}$ donating to two acceptors ${ }^{21,22}$ is not permitted to be a stable state, even though such structures are known to be present in x-ray crystal structures. ${ }^{83,84}$ It would be valuable if such a limitation could be removed.

In light of these criticisms, in this work we examine the nature of the non-tetrahedral components of water in a molecular dynamics simulation. The objectives are first of all to assess the validity of the topological HB classification and the resulting structure of water. Stability 
is assessed by the donor-acceptor distance $r_{\mathrm{OH}}$, the $\mathrm{HOO}$ angle $\theta_{\mathrm{HOO}}$ and the pairwise energy between two molecules $U_{\mathrm{ij}}$. We examine three influences on HB stability: the size of the closed HB polygons to which a HB belongs, the acceptor type, and the relative closeness of multiple donors to an acceptor. Secondly, the three assumed transition states are examined in more detail to determine if they really are transition states.

\section{Methods}

\subsection{Molecular Dynamics Simulations}

A cubic periodic box containing 1000 TIP4P/2005 water molecules ${ }^{94}$ and having volume approximately $3 \times 3 \times 3 \mathrm{~nm}^{3}$ is simulated using the sander module of the AMBER 12 software package. ${ }^{95} 500$ steps of steepest-descent minimisation are followed by a molecular dynamics simulation in the NVT ensemble at $298 \mathrm{~K}$ using a Langevin thermostat for $100 \mathrm{ps}$ in the NPT ensemble using a Berendsen barostat at 1 bar for a further 2 ns. Data collection proceeds over a further 1 ns under the same NPT conditions with SHAKE applied to all bonds, a 2 fs timestep, periodic boundary conditions, an $8 \AA$ cutoff and particle mesh Ewald with default AMBER parameters. Coordinates are saved every 1 ps for analysis.

\subsection{Hydrogen Bond Analysis}

HBs are analysed using the topological definition. ${ }^{31}$ The oxygen's charge of TIP4P/2005 is taken as the position of the acceptor as opposed to the mass and van der Waals centre as had been done in the original implementation based on distance. This is to be consistent with the general definition based on donor-acceptor force. ${ }^{31,44,96,97}$ As noted earlier, ${ }^{44}$ this makes little difference 
to the HB distribution, yielding marginally more broken HBs. For each HB coordination, we analyse the probability distributions in distance $r_{\mathrm{OH}}$ between the donor hydrogen and acceptor oxygen, the angle $\theta_{\mathrm{HOO}}$ subtended by the $\mathrm{HB} r_{\mathrm{OH}}$ vector and the donor's $\mathrm{HO}$ bond vector, and the interaction energy $U_{\mathrm{ij}}$ between the two molecules, calculated by summing over all electrostatic and Lennard-Jones energies of each atom pair. Three different comparisons are made:

1. We consider every donor and calculate the distributions of its nearest and next-nearest acceptors measured by the $r_{\mathrm{OH}}$ distance as a function of the number of acceptors of the nearest acceptor. We also examine these distributions according to whether the donor is a $\mathrm{BO}$ or $\mathrm{CD}$, and when the next-nearest acceptor does not have a $\mathrm{HB}$ with the donor molecule.

2. We consider the reverse situation by taking each acceptor and finding the series of nearest donors up to a maximum of the number of acceptors plus one. Again, the outermost donor that does not have a HB with the acceptor molecule is also analysed separately.

3. We consider how the HBs are strained by the constraints of the polygons to which they belong in the HB network. Polygons are defined using the algorithm of Chihaia et al. ${ }^{98}$ Different to previous work, ${ }^{80-82,98}$ dimers, comprising BOs and CDs, are also considered.

The probability distribution for each HB species is normalised to one. The exceptions are the distributions of neighbours which exclude a HB to the donor's water to make it easier to assess the effect of this exclusion. 


\subsection{Order Parameters}

In addition to the widely used tetrahedral order parameter, ${ }^{99}$ denoted here as $q_{4}$, two new analogous order parameters are considered: the trigonal and trigonal bipyramidal order parameters, denoted by $q_{3}$ and $q_{5}$, respectively. All three are calculated using the equations

$$
q_{3}=1-\frac{4}{7} \sum_{j=1}^{2} \sum_{k=j+1}^{3}\left(\cos \psi_{j k}+1 / 2\right)^{2}
$$

where the ideal angle is $120^{\circ}$,

$$
q_{4}=1-\frac{3}{8} \sum_{j=1}^{3} \sum_{k=j+1}^{4}\left(\cos \psi_{j k}+1 / 3\right)^{2}
$$

where the ideal angle is $109.5^{\circ}$, and

$$
q_{5}=1-\frac{6}{35} \sum_{i=1}^{2} \sum_{j=i+1}^{3}\left(\cos \psi_{i j}+1 / 2\right)^{2}-\frac{3}{10} \sum_{i=1}^{2} \sum_{k=1}^{3}\left(\cos \psi_{i k}\right)^{2}-\frac{3}{40}\left(\cos \psi_{k k}+1\right)^{2}
$$

where the ideal angles are $120^{\circ}$ for the three equatorial angles, $90^{\circ}$ for the six axial-equatorial angles, and $180^{\circ}$ for the one axial-axial angle. Indices $i$ and $j$ indicate equatorial positions and $k$ indicates axial positions, and the angles $\psi$ are calculated over all oxygen-oxygen-oxygen angles centred on the acceptor of consideration and involving the nearest three oxygens for $q_{3}$, four oxygens for $q_{4}$, and five oxygens for $q_{5}$. The reference angles could be chosen more accurately for the expected water geometry, such as having a tetrahedral value for the $\mathrm{HOH}$ angle instead of $120^{\circ}$, but a more symmetrical set was chosen for simplicity. The equations for $q_{3}$ and $q_{5}$ are derived using a similar method to that of Chau and Hardwick ${ }^{100}$ but normalised to one for perfect order and zero for a random distribution. ${ }^{99}$ The three equatorial oxygens in the case of $q_{5}$ are those whose angles between their $\mathrm{OO}$ vector and the water molecule's $\mathrm{HOH}$ plane are 
closest to zero. The remaining two are axial. Each order parameter is applied to SAs, DAs and TAs. They are normalised for each species so they can be more easily compared.

\subsection{Triple and Quadruple Acceptor Geometry}

Because the acceptor positions in TAs and QAs are non-equivalent, the probability distributions of the $\mathrm{OH}$ distances for the axial and equatorial donors to these two acceptors are calculated, together with those of the angles between their $\mathrm{OH}$ vectors and the water molecule's $\mathrm{HOH}$ plane, termed $\theta_{\mathrm{HOp}}$. The equatorial donor to a TA is determined as the one whose angle between its $\mathrm{OH}$ vector and the molecule's $\mathrm{HOH}$ plane is the closest to zero. Similarly for QAs, the two equatorial donors are those with these angles closest to zero. In each case the remaining two donors are axial.

\subsection{HB Switching Profiles}

The $\mathrm{HB}$ switching profiles via the three structures, $\mathrm{BH}, \mathrm{BO}$ and $\mathrm{CD}$, are plotted using the potential of mean force (PMF), defined as

$$
\mathrm{PMF}=-R T \ln P(\zeta)
$$

where $R$ is the universal gas constant, $T$ is temperature, $\zeta$ is the reaction coordinate and $P(\zeta)$ is its probability. Probabilities are normalised to one for all species present in each graph. Two choices of $\zeta$ are considered. They are designed such that $\zeta=0$ at the putative transition state. One is in terms of distance $\Delta r=\left|r_{\mathrm{OH} 1}-r_{\mathrm{OH} 2}\right|$ and the other in angle $\Delta \theta=\left|\theta_{\mathrm{HOO} 1}-\theta_{\mathrm{HOO} 2}\right|$. The subscript " 1 " designates the donor and its nearest acceptor for all transition states. "2" differs for each structure. For the BH it designates the donor and its next-nearest acceptor, for the BO it designates the donor molecule's other hydrogen and both donor's acceptor, and 
for the CD it designates the nearest donor of the donor molecule's acceptor and the donor molecule's oxygen. For the BO and CD structures, two specific cases of PMF are considered: one when the donor is broken and one when it is intact. For the BH structure, a whole series of PMFs are considered to examine the effects of three factors: the number of acceptors of the nearest and next-nearest acceptors, and the proximity of the donor to its acceptor. Proximity of a donor to an acceptor is assessed by assigning each donor to a subshell ranked according to $r_{\mathrm{OH}}$. The subshell ranges from 1 up to the number of acceptors for the closest to furthest donors, respectively.

\subsection{Donor-Acceptor Bias}

To detect the presence of any long-range structuring in terms of an imbalance of donors and acceptors, the cumulative donor-acceptor bias $\left.^{44,101} \Delta N_{(} r_{\mathrm{OO}}\right)$ is integrated from the origin to a distance $r_{\mathrm{OO}}$ from a water molecule:

$$
\Delta N\left(r_{\mathrm{OO}}\right)=\int_{0}^{r_{\mathrm{OO}}} \sum_{i}\left(d_{i}\left(r_{\mathrm{OO}}^{\prime}\right)-a_{i}\left(r_{\mathrm{OO}}^{\prime}\right)\right) d r^{\prime} O A
$$

where $d_{i}\left(r_{\mathrm{OO}}^{\prime}\right)$ and $a_{i}\left(r_{\mathrm{OO}}^{\prime}\right)$ are the numbers of donors and acceptors of water species $i$ at distance $r_{\mathrm{OO}}^{\prime}$ from each water.

\section{Results}

\subsection{Hydrogen Bond Analysis}

The HB distributions produced by the topological definition are $0.2 \% \mathrm{NA}, 15.0 \% \mathrm{SA}, 71.5 \% \mathrm{DA}$, $13.0 \%$ TA, $0.3 \%$ QA, $0.0 \%$ non-donor, $0.3 \%$ BO, $1.5 \% \mathrm{CD}$, and $98.2 \%$ double donor at $298 \mathrm{~K}$ 
and 1 bar as reported before. ${ }^{31}$ Similar populations are produced using other force fields ${ }^{31}$ and from ab initio simulation, although there is a dependence on the size of the dispersion term. ${ }^{70}$ Given that BOs are responsible for about half as many HB switches as CDs,${ }^{31}$ the much smaller percentrage of BOs implies that the reaction coordinate for switching via a $\mathrm{BO}$ must be shorter than that via a $\mathrm{CD}$. This is consistent with a $\mathrm{BO}$ switch requiring the rotation of only one molecule through the tetrahedral angle whereas the CD switch requires the rotation of two molecules through the tetrahedral angle.

Figure 1 plots the probability distributions in $r_{\mathrm{OH}}, \theta_{\mathrm{HOO}}$ and $U_{\mathrm{ij}}$ of a donor's nearest acceptor and next-nearest acceptor for each acceptor species and for the broken donor of the BO and CD species. NAs are not included because by definition they are almost never a donor's preferred acceptor. They are only ever so when the NA's donor HB back to the molecule is stronger, leading to a broken HB to the NA. The distributions for QAs and the broken HB species are slightly noisier because of the much lower populations of these species. The main result is that that data support the assumption that a donor donates strongly to its nearest acceptor. The values of distance, angle or energy are low for the nearest acceptor, and there is a large difference going to the next-nearest acceptor. This demonstrates that a donor's HB to the nearest acceptor is usually much stronger than its HB to the next-nearest acceptor. This distinction is sharpest for the species with fewer acceptors which are able to accept HBs more optimally because there are fewer constraints and less crowding brought about by other donors. It is also sharpest for the distance distributions of SAs, DAs and TAs, for which the combined nearest and next-nearest distributions show a well-defined minimum. The stability of the donors to QAs is marginal. It is inevitable that there is some overlap between the nearest and next-nearest peaks because the donors exchange and spend some time at intermediate distances. The distributions for donors having broken HBs in BOs and CDs resemble those of the next-nearest acceptors, signifying much weaker donor-acceptor interactions as expected. The double-hump structure evident in 
the angle and energy distributions of the next-nearest acceptors can be understood as arising from two types of water molecules: those already in the first hydration shell with another HB to the donor and those without. This is made clear by the plots which exclude other first-shell water molecules in the next-nearest distribution. They have similar distances but lower angles peaked around $50^{\circ}$ and energies much closer to zero $\mathrm{kJ} \mathrm{mol}^{-1}$, consistent with them not being in the first hydration shell.

Next we turn to Figure 2 which plots the probability distributions in $r_{\mathrm{OH}}, \theta_{\mathrm{HOO}}$ and $U_{\mathrm{ij}}$ for the series of nearest donors for each type of acceptor. The main result here is that it supports the assignment of different HB coordinations in the first hydration shell. It would be farfetched to conclude that the nearest two donors are always within the first hydration shell and that no others are, as would be expected for tetrahedral coordination. Generally, the largest transition from one donor to the next occurs when going to the donor number that exceeds the species' number of acceptors. This is especially so for the angles, indicating that there is much directionality for the HBs, even up to the fourth-nearest HB of QAs. As seen in Figures 1 and 2, the HBs for species with more acceptors have longer distances, larger angles and higher energies, with the spacing increasing in size with acceptor number. The nearest donor outside the coordination shell is either near the tetrahedral angle, suggesting that it already donates to the acceptor, or it lies in a broad distribution centred at $50^{\circ}$ which are weakly interacting water molecules as noted before. The equivalent distributions using traditional HB definitions with a cut-off in $r_{\mathrm{OH}}^{55}$ or energy ${ }^{18,58-60}$ would invariably have a sharp discontinuity at the cut-off. Such sharp transitions are softened in these one-dimensional projections for definitions with two or more more criteria such as the those based on a distance and angle ${ }^{49,53,54,56,57}$ because each variable is coupled. Nevertheless, a sharp discontinuity would still occur in the full, higher-dimensional probability distribution. These features would be absent using the nearest-acceptor and two-nearest-donors definition ${ }^{68}$ but the TA and QA species would be 
entirely absent because only a maximum of two acceptors are allowed per water molecule.

The third factor examined to assess HB strength and in particular, broken HBs, is the effect of the polygon ring size to which a HB belongs. The same distance, angle and energy distributions are plotted in Figure 3. We do not consider the many permutations of HB directions in the ring, ${ }^{17}$ as had been done for the $\mathrm{BO}$ and $\mathrm{CD}$ structures before in Figure 1. Moreover, we suspend here the assignment of broken HBs brought about by the dimers. It can be seen that trimers induce a moderate amount of distortion, especially for the weakest HB. The distributions for the tetramers and pentamers are almost indistinguishable and show a gradual increase in strain from the strongest to the weakest $\mathrm{HB}$, suggesting that rings of these sizes induce negligible strain. These results support a possible case for considering the weakest $\mathrm{HB}$ in a trimer as broken. The number of trimers calculated per water molecule is 0.017 , comparable to the 0.018 for dimers. Thus there would be twice as many broken HBs if trimer distortion were also regarded as leading to a broken HB. This makes clear that the distinction between broken and intact HBs is less distinct, even by the topological definition. The different levels of distortion are discrete for the smallest polygons but become more continuously distributed for larger polygons.

\subsection{Order Parameters}

As another measure of coordination, the trigonal, tetrahedral and trigonal bipyramidal order parameters are plotted in Figures 4(a)-(c) for SAs, DAs, and TAs. Each parameter is largest for its respective species, confirming that their geometries mostly match that of the appropriate order parameter. In particular, the SAs and TAs are seen to be responsible for the shoulder in the $q_{4}$ plots that are typically rationalised as being less tetrahedral. The only species which are not well distinguished are the SAs and DAs by the $q_{3}$ order parameter. This indicates that 
the nearest three waters for tetrahedral coordination are often very close to trigonal.

\subsection{Triple and Quadruple Acceptor Geometry}

To examine further the donor distributions around the acceptors with more than one donor type, namely TAs and QAs, Figures 5(a)-(d) illustrate that the axial and equatorial $\mathrm{OH}$ distances and donor angles to the plane $\theta_{\mathrm{HOp}}$ are very similar. Axial distances are marginally shorter by $\sim 0.01 \mathrm{~nm}$ than equatorial ones, TA's equatorial donor is more in the plane than the QA ones, and the angles of QA's axial donors are marginally more axial than the TA ones. All these trends are consistent with the expected geometries.

\subsection{HB Switching Profiles}

Here we assess whether the $\mathrm{BH}, \mathrm{BO}$ and $\mathrm{CD}$ structures are transition states. Figure 6 illustrates the donor-acceptor PMFs for each of the three cases. The transition states lie where the value of the reaction coordinate equals zero. The plots are not continued beyond this point because they are symmetric about zero. All the PMF curves display a maximum at the origin. This would appear to validate the assumption made in the topological definition and elsewhere ${ }^{20,23-26,28,29,50}$ that these geometries are transition states. Another observation is that there is a high barrier near the transition state for the $\mathrm{BO}$ and $\mathrm{CD}$ PMFs when the donor HB is still intact. Once the donor HB has broken, the barrier for switching becomes much lower and both these structures are more delocalised, facilitating the switch.

Now we examine the more detailed PMFs in terms of the number of acceptors of a donor's nearest and next-nearest acceptor as well as the donor's subshell to its nearest acceptor. These PMFs are displayed in Figure 7 as a function of $\Delta r$ or $\Delta \theta$. The plots are not extended to 
negative values of the reaction coordinate because the acceptor types would change for $\Delta r$. What these PMFs quantify is how easy it is for a donor to move from a given subshell of one acceptor type to another acceptor type. A much more intricate and intriguing picture emerges than what was seen in Figure 6. In the outermost subshell of DAs and TAs, it can be seen that the barrier for switching to a lower-acceptor species is essentially removed! To consider all the possible cass, we define the acceptor differential $\Delta a$ as the number of acceptors of the next-nearest acceptor minus that of the nearest acceptor. The greater $\Delta a$, the more favourable becomes the PMF for a switch. For switches with $\Delta a=-1$, such as a DA to a SA or a TA to a DA, the reactant and product states are identical by symmetry. Such a situation is the hallmark of a stable $\mathrm{BH}$, as proposed by Giguère ${ }^{21,22}$ The percentage of donors existing as a $\mathrm{BH}$ can be estimated to be $10 \%$ by taking the $15 \%$ and $13 \%$ of SAs and TAs, converting them into $7.5 \%$ and $6.5 \%$ of the outer-subshell donors, and noting that their neighbour would be a DA about $70 \%$ of the time. This number may seem large but it includes asymmetric BH structures as well as the more symmetric ones. For switches with $\Delta a<-2$, such as when a donor switches from a TA to a SA, there is no barrier and a continual decrease in PMF that would lead to a more stable product state. For switches with $\Delta a \geq 0$, then the switch is unfavourable. The most common such switch is two DAs going to a SA and TA. It nonetheless proceeds because of the high concentration of DA.

These results may be surprising in light of the predominance of work suggesting that BHs are transition states, ${ }^{20,23-25,28,29,50}$ However, they become more intuitive upon further reflection. The fewer donors an acceptor has, the easier it is for another donor to donate to it and vice versa. What is noteworthy is that this trend only applies to donors in an acceptor's outermost subshell. For other types of switch, the barriers are substantially higher. Evidently a donor that is interacting more strongly with its acceptor is less likely to leave. This emphasises the point that if the different subshells had not been considered individually, then these effects would 
have been missed and barriers would have appeared for all acceptor types. In any case, rapid whole-molecule vibration continually changes the ranking of the nearest donors to an acceptor, giving each donor in turn a chance to switch. Interestingly, the lifetimes of SAs and TAs, determined earlier to be about $\sim 0.3 \mathrm{ps}$, match the timescale of whole-molecule vibration. ${ }^{31}$ We do not need to consider the ranking of a donor to its next-nearest acceptor because it should automatically be further out than any of this acceptor's donors. The final piece in the mechanism is to recognise that because a donor's preferred position depends upon $\Delta a$ and on its ranking to its nearest acceptor, when either of its two nearest neighbours change acceptor number or when its ranking to its acceptor changes, then so does the donor's preferred position. For example, consider a donor pointing to a DA with another DA nearby. As long as this donor is the outermost donor to its DA, if this nearby DA becomes an SA, then the donor becomes bifurcated between the two. If the nearby SA drops to a NA, then the bifurcated donor switches entirely to the NA, making it a full SA. This demonstrates how switches are fully at the mercy of their neighbours' switches in a complex recursive fashion.

The implications of these findings are profound. Firstly, it reveals that the nature of nontetrahedral water is quite different to what is usually envisaged. Its identity is intimately coupled to its nearest neighbours, sometimes with a donor directed to an acceptor and sometimes bifurcated. In particular, it clarifies that BHs may be caused by either SAs or TAs but explains why not all SAs or TAs lead to BHs. It is also understandable why these non-tetrahedral structures with their donor-acceptor imbalances are largely resistant to quenching. ${ }^{31}$ Furthermore, drawing on HB structures as precursors to proton transfer, ${ }^{71}$ the bifurcated forms of the SAs and TAs bear striking resemblances to Zundel forms of the hydrated proton ${ }^{90}$ and hydroxide ion. ${ }^{91}$ Secondly, a question mark is raised about the usefulness of the topological HB definition whose main assumption is that the donor donates to its nearest acceptor. The cancellation of the forces on the donor from two different acceptors may approximate a nearby stationary 
point but it turns out to be a minimum rather than a transition state as had been initially assumed. Consequently, a more sophisticated procedure is required to allow for the possibility of a BH. The definition would need the qualification that as well as donating to its nearest acceptor, a donor may also donate to its second-nearest acceptor if it is the outermost donor to its closest acceptor and if the second-nearest acceptor has fewer acceptors than the nearest acceptor! This wordy definition reflects the greater complexity of HB structure in water. The presence of the BHs also makes the classification of the species by donor and acceptor number alone more complex. One might have a partial DA, for example, when one of the donors to it is bifurcated. Thirdly, HB switches via a BH for an acceptor's outermost donor are either uphill, downhill, or largely flat. There is effectively no barrier for this type of switch. It is the combination of all of these processes as seen in Figure 6 that creates the illusion of a barrier, particularly the contribution from the more stable, inner subshells that do have a substantial barrier. Inner subshell donors can only switch if the outer donor, being further away, is also switching. Such barrierless donor switching draws an unavoidable parallel with the barrierless Grotthuss mechanism of proton transfer. ${ }^{90}$ In both cases, it is the HB defects in the surrounding water network that determines what switches can take place rather than the switch itself. Fourthly, BHs lead on average to more than four HBs per molecule in its first coordination shell and contribute to water's OO coordination number being 4-5. Fifthly, BHs even have the effect of making water structure look more tetrahedral. SAs are supplemented by a BH and one of the TA's HBs is weaker and bifurcated. Even so, the non-tetrahedral structures not engaged in BHs persist.

\subsection{Donor-Acceptor Bias}

The final question to be examined, and incidentally the original objective of this work, is whether there is long-range structure in water. The measure for this considered here is the 
cumulative donor-acceptor bias $\left.\Delta N_{(} r_{\mathrm{OO}}\right)$ which is plotted in Figure 8. While there is an imbalance at short-range, with initially fewer donors than acceptors at a distance corresponding to the minimum in the first hydration shell, this trend reverses further away and oscillates with decreasing amplitude, reaching zero comfortably within half the box-length. Thus there is no long-range structuring of water by this measure. Similar results for the distribution of individual HB species ${ }^{44}$ also turn up similar null long-range results (data not shown). This is not surprising, given that water on average has the same number of donors and acceptors with itself. Nevertheless, it still needed to be confirmed. There is certainly structuring at short-range in water, with tetrahedral water displaying a slight preference for itself, as is well-known, ${ }^{18,47}$ and high and low acceptor species such as TAs and SAs tending to attract each other more than average to alleviate imbalances in donors and acceptors. ${ }^{31}$ However, these biases decay rapidly with distance beyond a few hydration shells. Thus it can be concluded that the presence of a solute or interface must be required to bring about long-range structuring in water, ${ }^{44}$ at least in the type of structuring considered here. Such long-range structuring occurs because the the short-range perturbation induced by the solute or interface in the donor-acceptor balance of water's HBs is best accommodated by spreading the compensating donor-acceptor balance over as many water molecules as possible. This capability of water presumably contributes to its ability to solvate molecules. The distribution of HB species with different numbers of donors and acceptors as proposed here would be essential in accommodating the solute's HB preferences and present interesting possibilities at many levels of structure.

\section{Conclusions}

The case for non-tetrahedral water has been put forward, based primarily on the greater reliability of the topological HB definition which resolves HBs based on the transition states between 
them rather than using cut-off-based HB definitions with their unavoidable ambiguities. This is bolstered here by new evidence from molecular dynamics simulations which confirms the identities of the non-tetrahedral components. This evidence includes distance, angle and energy distributions, new order parameters for the trigonal and trigonal bipyramidal coordinations, and an analysis on the HB distortion induced by closed HB polygons. However, in seeking to verify the positions of these transition states, it was found that $\mathrm{HB}$ switching via the $\mathrm{BH}$ is largely a barrierless process. The change in acceptor number going from the nearest to the nextnearest acceptor controls the likelihood of a switch's PMF, as does the subshell of the donor to its acceptor. In particular, the lower the acceptor number of the next-nearest acceptor, and the further away the donor is from its acceptor, the more likely the switch. This is in contrast to most studies which imply that the $\mathrm{BH}$ is a transition state. While no long-range structuring was detected in water in terms of a donor-acceptor imbalance, the non-tetrahedral species presented here are expected to play a major role in long-range structuring around dissolved solutes, as has already been seen. ${ }^{44}$ Such structuring efficiently dissipates the perturbation induced by the solute over the rest of the water. The ramifications of this new picture of water may be equally far-reaching.

\section{Acknowledgments}

We are indebted to E Arunan, G Desiraju, Prabal Maiti, Noam Agmon, Raphael Levine, Mark Tuckerman, John Finney, Imre Bako, David Chandler, Frank Stillinger, Frauke Gräter, Ulf Hensen, Anupam Chatterjee and Sheeba Jem Irudayam for helpful discussions about hydrogen bonds and water structure, not to mention instructive comments by the anonymous reviewer. This work was carried out with support from the Alexander von Humboldt Foundation, the German Academic Exchange Service, the Klaus Tschira Foundation, the Lady Davis Fellow- 
ship Trust, the Indian Institute of Science, the EPSRC-funded Manchester Chemical Biology Network (EP/I037253/1), and BBSRC grant BB/K001558/1. 


\section{References}

1. A. H. Narten and H. A. Levy, Science, 1969, 165, 447-454.

2. F. H. Stillinger, Science, 1980, 209, 451-457.

3. A. K. Soper and M. G. Phillips, Chem. Phys., 1986, 107, 47-60.

4. J. D. Bernal and R. H. Fowler, J. Chem. Phys., 1933, 1, 515-548.

5. J. A. Pople, Proc. R. Soc. London Ser. A, 1951, 205, 163-178.

6. M. G. Sceats and S. A. Rice, J. Chem. Phys., 1980, 72, 6183-6191.

7. Y. Marechal, The hydrogen bond and the water molecule, Elsevier, Oxford, 2007.

8. A. K. Soper, Pure Appl. Chem., 2010, 82, 1855-1867.

9. G. N. I. Clark, C. D. Cappa, J. D. Smith, R. J. Saykally, and T. Head-Gordon, Mol. Phys., 2010, 108, 1415-1433.

10. I. M. Svishchev and P. G. Kusalik, Chem. Phys. Lett., 1993, 215, 596-600.

11. P. G. Kusalik and I. M. Svishchev, Science, 1994, 265, 1219-1221.

12. J. D. Smith, C. D. Cappa, K. R. Wilson, R. C. Cohen, P. L. Geissler, and R. J. Saykally, Proc. Natl. Acad. Sci. U.S.A., 2005, 102, 14171-14174.

13. S. Dixit, J. Crain, W. C. K. Poon, J. L. Finney, and A. K. Soper, Nature, 2002, 416, 829-832.

14. W. K. Röntgen, Ann. Phys., 1892, 281, 91-97.

15. D. Eisenberg and W. Kauzmann, The structure and properties of water, Oxford University Press, Oxford, 1969.

16. R. Ludwig, Angew. Chem. Int. Edit., 2001, 40, 1808-1827.

17. G. Malenkov, J. Phys.: Condens. Matter, 2009, 21, 283101.

18. H. E. Stanley and J. Teixeira, J. Chem. Phys., 1980, 73, 3404-3422.

19. C. Huang, K. T. Wikfeldt, T. Tokushima, D. Nordlund, Y. Harada, U. Bergmann, M. Niebuhr, T. M. Weiss, Y. Horikawa, M. Leetmaa, M. P. Ljungberg, O. Takahashi, A. Lenz, L. Ojamae, A. P. Lyubartsev, S. Shin, L. G. M. Pettersson, and A. Nilsson, Proc. Natl. Acad. Sci. U.S.A., 2009, 106, 15214-15218.

20. J. D. Eaves, J. J. Loparo, C. J. Fecko, S. T. Roberts, A. Tokmakoff, and P. L. Geissler, Proc. Natl. Acad. Sci. U.S.A., 2005, 102, 13019-13022. 
21. P. A. Giguère, J. Raman Spectrosc., 1984, 15, 354-359.

22. P. A. Giguère, J. Chem. Phys., 1987, 87, 4835-4839.

23. F. Sciortino, A. Geiger, and H. E. Stanley, Phys. Rev. Lett., 1990, 65, 3452-3455.

24. F. Sciortino, A. Geiger, and H. E. Stanley, Nature, 1991, 354, 218-221.

25. F. Sciortino, A. Geiger, and H. E. Stanley, J. Chem. Phys., 1992, 96, 3857-3865.

26. A. Geiger, M. Kleene, D. Paschek, and A. Rehtanz, J. Mol. Liq., 2003, 106, 131-146.

27. N. Grishina and V. Buch, Chem. Phys. Lett., 2003, 379, 418-426.

28. D. Laage and J. T. Hynes, Science, 2006, 311, 832-835.

29. D. Laage and J. T. Hynes, J. Phys. Chem. B, 2008, 112, 14230-14242.

30. O. Markovitch and N. Agmon, Mol. Phys., 2008, 106, 485-495.

31. R. H. Henchman and S. J. Irudayam, J. Phys. Chem. B, 2010, 114, 16792-16810.

32. G. Nemethy and H. A. Scheraga, J. Chem. Phys., 1962, 36, 3382-3400.

33. I. Ohmine, J. Phys. Chem., 1995, 99, 6767-6776.

34. A. Botti, F. Bruni, M. A. Ricci, and A. K. Soper, J. Chem. Phys., 1998, 109, 3180-3184.

35. M. Bernabei, A. Botti, F. Bruni, M. A. Ricci, and A. K. Soper, Phys. Rev. E, 2008, 78, 021505.

36. H. S. Frank and M. W. Evans, J. Chem. Phys., 1945, 13, 507-532.

37. J. C. Owicki and H. A. Scheraga, J. Am. Chem. Soc., 1977, 99, 7403-7412.

38. S. Swaminathan, S. W. Harrison, and D. L. Beveridge, J. Am. Chem. Soc., 1978, 100, 5705-5712.

39. S. J. Irudayam and R. H. Henchman, J. Phys.: Condens. Matter, 2010, 22, 284108.

40. J. G. Davis, K. P. Gierszal, P. Wang, and D. Ben-Amotz, Nature, 2012, 491, 582-585.

41. N. Galamba, J. Phys. Chem. B, 2013, 117, 589-601.

42. G. R. Desiraju, Chem. Comm., 1991, pp. 426-428.

43. L. Infantes, L. Fabian, and W. D. S. Motherwell, Crystengcomm, 2007, 9, 65-71.

44. S. J. Irudayam and R. H. Henchman, J. Chem. Phys., 2012, 137, 034508.

45. P. Ball, Chem. Rev., 2008, 108, 74-108. 
46. Y. Marcus, Chem. Rev., 2009, 109, 1346-1370.

47. H. S. Frank and W. Y. Wen, Discuss. Faraday Soc., 1957, 24, 133-140.

48. M. Falk and T. A. Ford, Can. J. Chem., 1966, 44, 1699-1707.

49. P. Wernet, D. Nordlund, U. Bergmann, M. Cavalleri, M. Odelius, H. Ogasawara, L. A. Naslund, T. K. Hirsch, L. Ojamae, P. Glatzel, L. G. M. Pettersson, and A. Nilsson, Science, 2004, 304, 995-999.

50. S. T. Roberts, K. Ramasesha, and A. Tokmakoff, Accounts Chem. Res., 2009, 42, 12391249 .

51. T. D. Kühne and R. Z. Khaliulin, Nature Commun., 2013, 4, 1450-1456.

52. N. Agmon, Accounts Chem. Res., 2012, 45, 63-73.

53. A. C. Belch, S. A. Rice, and M. G. Sceats, Chem. Phys. Lett., 1981, 77, 455-459.

54. M. Mezei and D. L. Beveridge, J. Chem. Phys., 1981, 74, 622-632.

55. V. Buch, J. Chem. Phys., 1992, 96, 3814-3823.

56. A. Luzar and D. Chandler, Phys. Rev. Lett., 1996, 76, 928-931.

57. R. Kumar, J. R. Schmidt, and J. L. Skinner, J. Chem. Phys., 2007, 126, 204107.

58. A. Rahman and F. H. Stillinger, J. Chem. Phys., 1971, 55, 3336-3359.

59. P. J. Rossky and M. Karplus, J. Am. Chem. Soc., 1979, 101, 1913-1937.

60. W. L. Jorgensen, Chem. Phys. Lett., 1980, 70, 326-329.

61. D. C. Rapaport, Mol. Phys., 1983, 50, 1151-1162.

62. G. Malenkov, D. Tytik, and E. Zheligovskaya, J. Mol. Liq., 1999, 82, 27-38.

63. H. F. Xu, H. A. Stern, and B. J. Berne, J. Phys. Chem. B, 2002, 106, 2054-2060.

64. L. Pauling, The nature of the chemical bond, Cornell, Ithaca, 3rd ed., 1960.

65. Q. Du, E. Freysz, and Y. R. Shen, Science, 1994, 264, 826-828.

66. G. Desiraju, Angew. Chem. Int. Edit., 2011, 50, 52-59.

67. F. H. Stillinger and T. A. Weber, Phys. Rev. A, 1982, 25, 978-989.

68. A. D. Hammerich and V. Buch, J. Chem. Phys., 2008, 128, 111101.

69. R. F. W. Bader, Chem. Rev., 1991, 91, 893-928.

70. Z. H. Ma, Y. L. Zhang, and M. E. Tuckerman, J. Chem. Phys., 2012, 137, 044506. 
71. E. Arunan, G. R. Desiraju, R. A. Klein, J. Sadlej, S. Scheiner, I. Alkorta, D. C. Clary, R. H. Crabtree, J. J. Dannenberg, P. Hobza, H. G. Kjaergaard, A. C. Legon, B. Mennucci, and D. J. Nesbitt, Pure Appl. Chem., 2011, 83, 1637-1641.

72. K. Morokuma and L. Pedersen, J. Chem. Phys., 1968, 48, 3275-3282.

73. P. A. Kollman and L. C. Allen, J. Chem. Phys., 1969, 51, 3286-3293.

74. T. R. Dyke, K. M. Mack, and J. S. Muenter, J. Chem. Phys., 1977, 66, 498-510.

75. F. N. Keutsch and R. J. Saykally, Proc. Natl. Acad. Sci. U.S.A., 2001, 98, 10533-10540.

76. N. Bjerrum, Science, 1952, 115, 385-390.

77. R. Podeszwa and V. Buch, Phys. Rev. Lett., 1999, 83, 4570-4573.

78. B. Mukherjee., P. K. Maiti, C. Dasgupta, and A. K. Sood, J. Phys. Chem. B, 2009, 113, 10322-10330.

79. J. J. Loparo, C. J. Fecko, J. D. Eaves, S. T. Roberts, and A. Tokmakoff, Phys. Rev. B, 2004, 70, 180201.

80. A. Rahman and F. H. Stillinger, J. Am. Chem. Soc., 1973, 95, 7943-7948.

81. A. C. Belch and S. A. Rice, J. Chem. Phys., 1987, 86, 5676-5682.

82. P. E. Mason and J. W. Brady, J. Phys. Chem. B, 2007, 111, 5669-5679.

83. M. Falk and O. Knop in Water: A Comprehensive Treatise, ed. F. Franks; Plenum Press, 1973; pp. 55-113.

84. G. A. Jeffrey and W. Saenger, Hydrogen bonding in biological structures, Springer, Berlin, 1991.

85. I. Olovsson and P. Jonsson in The hydrogen bond. Recent developments in theory and experiments, ed. P. Schuster, Z. G., and C. Sandorfy; North Holland, 1976; pp. 393-456.

86. G. F. Diercksen, Theor. Chim. Acta, 1971, 21, 335-367.

87. K. Hermansson, The electron distribuition in the bound water molecule., PhD thesis, University of Uppsala, Sweden, 1984.

88. J. L. Finney, J. E. Quinn, and J. O. Baum in Water Science Reviews, ed. F. Franks; Cambridge University Press, 1985; pp. 93-170.

89. J. L. Finney, Philos. T. Roy. Soc. B, 2004, 359, 1145-1163.

90. N. Agmon, Chem. Phys. Lett., 1995, 244, 456-462.

91. M. E. Tuckerman, D. Marx, and M. Parrinello, Nature, 2002, 417, 925-929. 
92. M. Goswami and E. Arunan, Phys. Chem. Chem. Phys., 2009, 11, 8974-8983.

93. G. Desiraju and T. Steiner, The weak hydrogen bond, Oxford University Press, Oxford, 1999.

94. J. L. F. Abascal and C. Vega, J. Chem. Phys., 2005, 123, 234505.

95. D. A. Case, T. A. Darden, T. E. Cheatham III, C. I. Simmerling, J. Wang, R. E. Duke, R. Luo, K. M. Merz, D. A. Pearlman, M. Crowley, R. C. Walker, W. Zhang, B. Wang, S. Hayik, A. Roitberg, G. Seabra, K. F. Wong, F. Paesani, X. Wu, S. Brozell, V. Tsui, H. Gohlke, L. Yang, C. Tan, J. Mongan, V. Hornak, G. Cui, P. Beroza, H. Mathews, D, C. Schafmeister, W. S. Ross, and P. A. Kollman, AMBER 12, University of California, San Francisco, 2012.

96. S. J. Irudayam, R. D. Plumb, and R. H. Henchman, Faraday Discuss., 2010, 45, 467-485.

97. S. J. Irudayam and R. H. Henchman, Mol. Phys., 2011, 109, 37-48.

98. V. Chihaia, S. Adams, and W. F. Kuhs, Chem. Phys., 2005, 317, 208-225.

99. J. R. Errington and P. G. Debenedetti, Nature, 2001, 409, 318-321.

100. P. L. Chau and A. J. Hardwick, Mol. Phys., 1998, 93, 511-518.

101. R. Vacha, O. Marsalek, A. P. Willard, D. J. Bonthuis, R. R. Netz, and P. Jungwirth, J. Phys. Chem. Letters, 2012, 3, 107-111. 


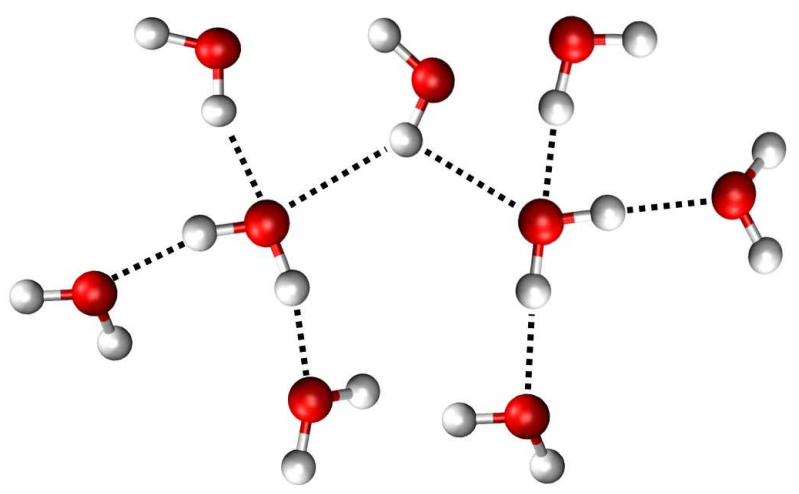

Transition state or stable? 

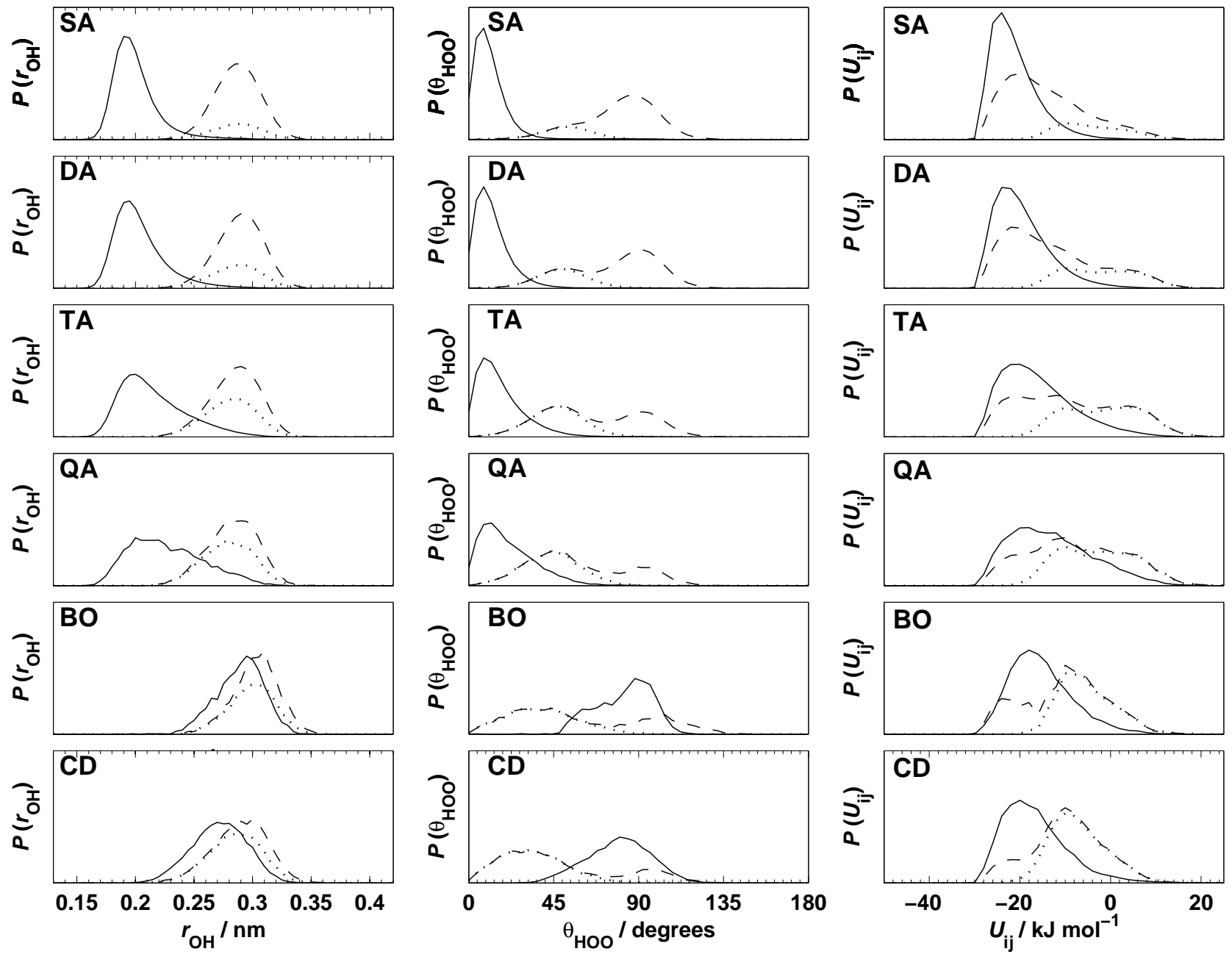

Figure 1: Probability distributions $P\left(r_{\mathrm{OH}}\right)$ of the $\mathrm{OH}$ distance $r_{\mathrm{OH}}$ from a donor to its nearest (solid) acceptor, $P\left(\theta_{\mathrm{HOO}}\right)$ of the $\mathrm{HOO}$ angle $\theta_{\mathrm{HOO}}$ bisected by the donor-acceptor vector and the donor's $\mathrm{OH}$ bond vector, and $P\left(U_{\mathrm{ij}}\right)$ of the pairwise energy $U_{\mathrm{ij}}$ of the donor-acceptor pair of water molecules. The acceptor is either a SA, $\mathrm{DA}, \mathrm{TA}, \mathrm{QA}, \mathrm{BO}$ or CD (see text for abbreviations). Also plotted are the values for the donor's next-nearest acceptor (dashed) and the next-nearest acceptor without a HB with the donor molecule (dotted). 

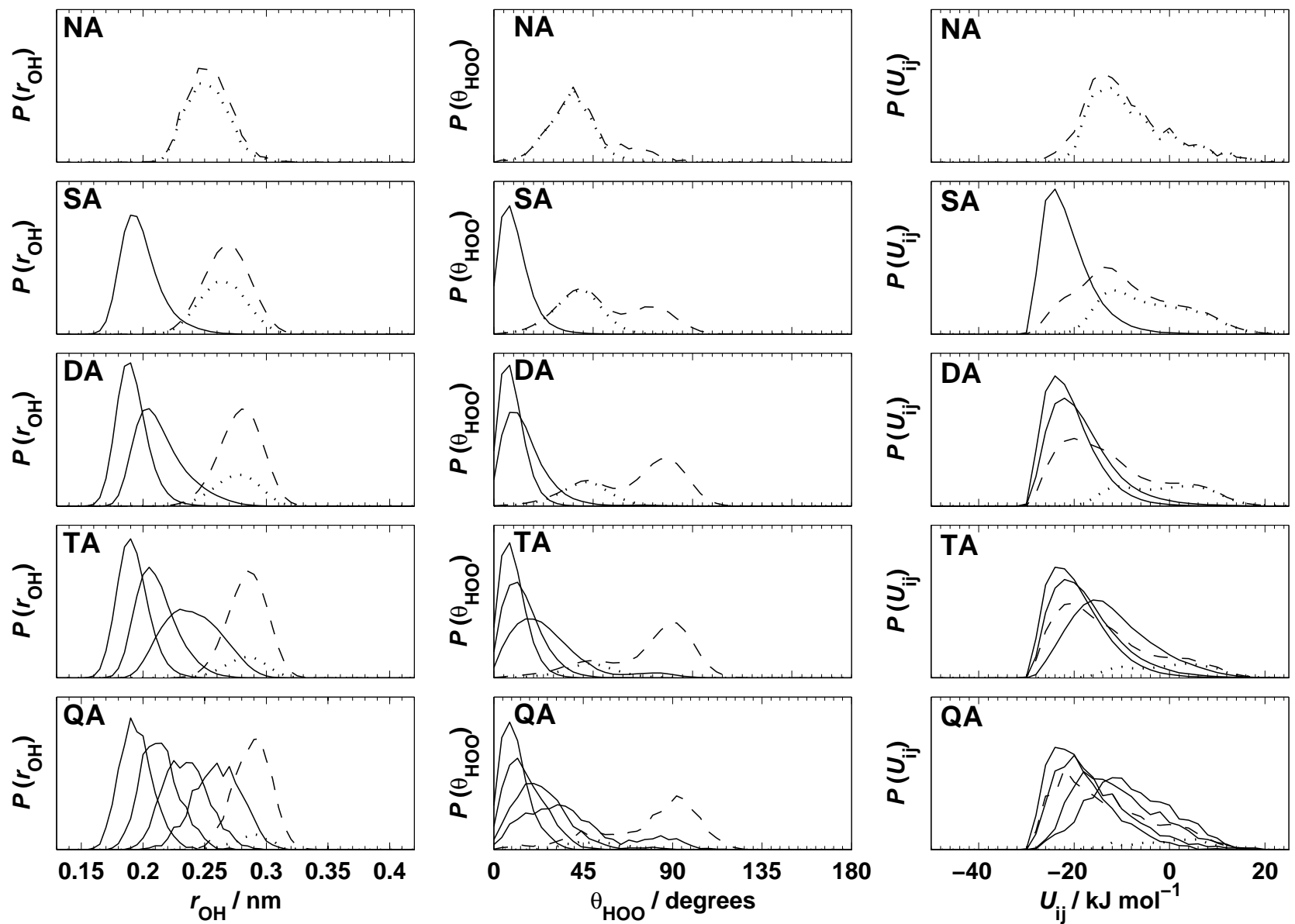

Figure 2: Probability distributions $P\left(r_{\mathrm{OH}}\right), P\left(\theta_{\mathrm{HOO}}\right)$ and $P\left(U_{\mathrm{ij}}\right)$ from an acceptor to its nearest, expected number of donors (solid). The distributions are ranked according to $r_{\mathrm{OH}}$. The acceptor is either a NA, SA, DA, TA, or QA. Also plotted are the values for the next-nearest donor beyond the expected acceptor number for that species (dashed) and the next-nearest donor that has no HB to the acceptor molecule (dotted). 

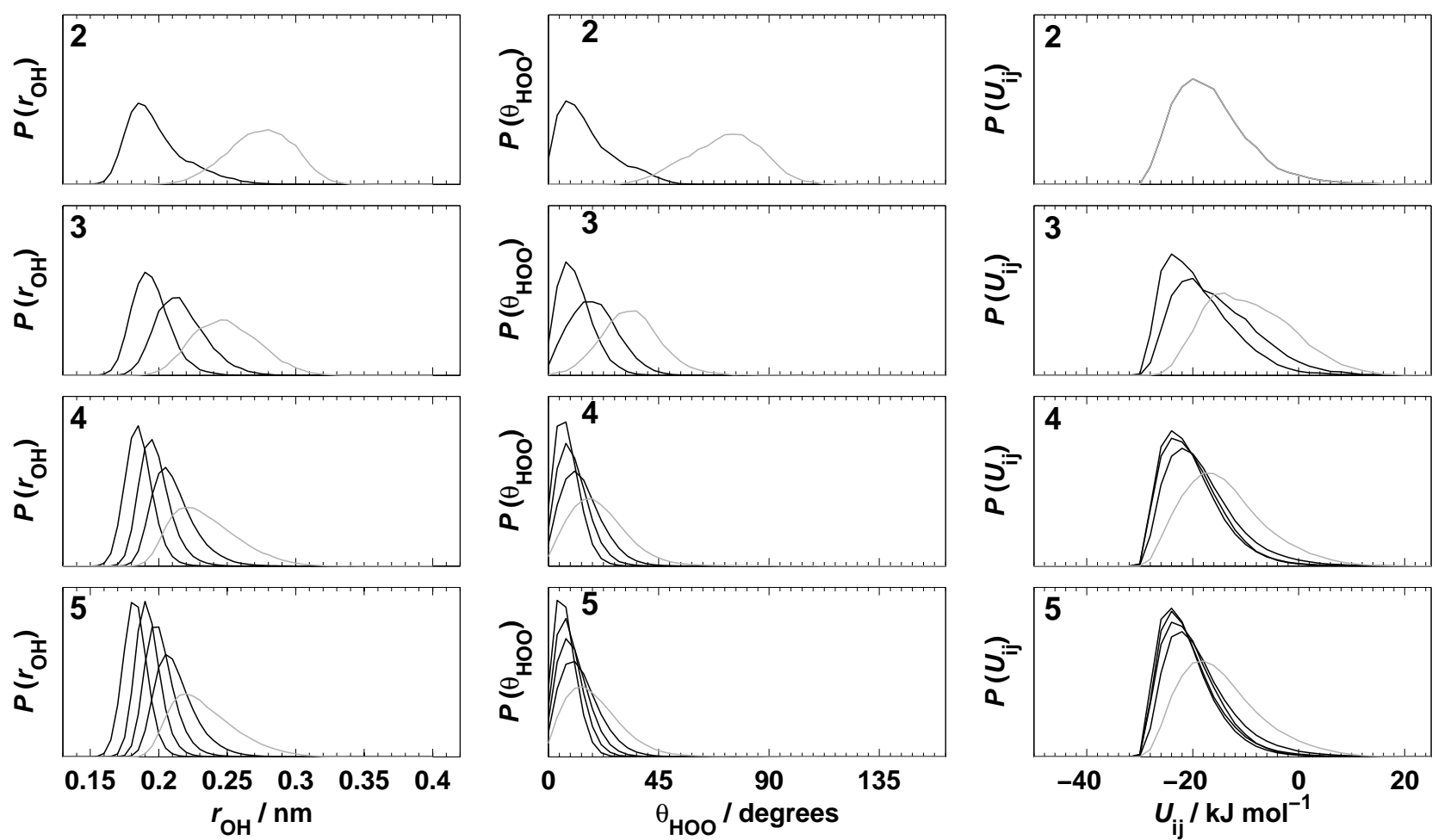

Figure 3: Probability distributions $P\left(r_{\mathrm{OH}}\right), P\left(\theta_{\mathrm{HOO}}\right)$ and $P\left(U_{\mathrm{ij}}\right)$ for all $\mathrm{HB}$ interactions in a polygon (solid). The polygons have sides from two to five. All distributions are ranked by $r_{\mathrm{OH}}$, and the weakest donor-acceptor distribution is shaded grey.
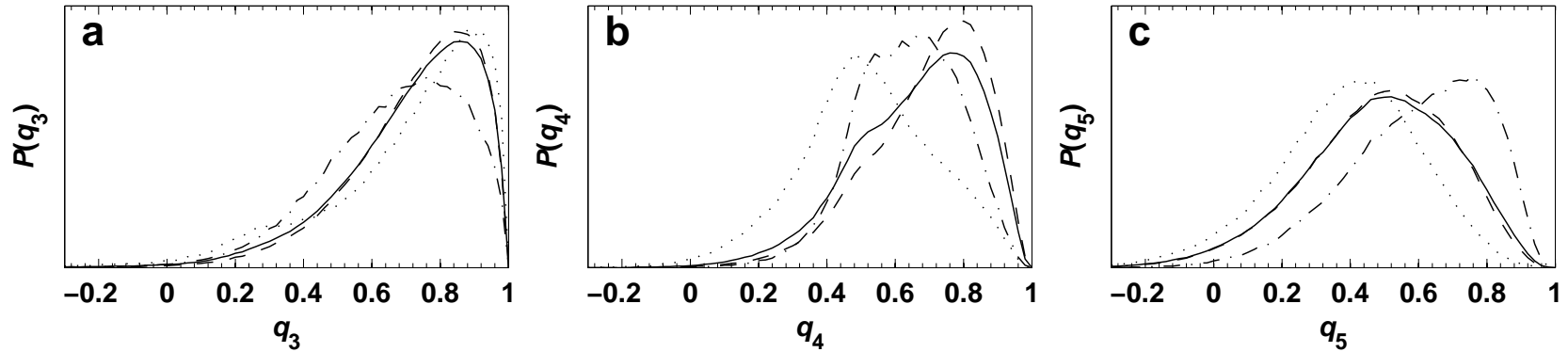

Figure 4: (a) Trigonal $q_{3}$, (b) tetrahedral $q_{4}$ and (c) trigonal bipyramidal $q_{5}$ order parameters applied to SAs (dotted), DAs (dashed), TAs (dot-dashed) and all water (solid). 

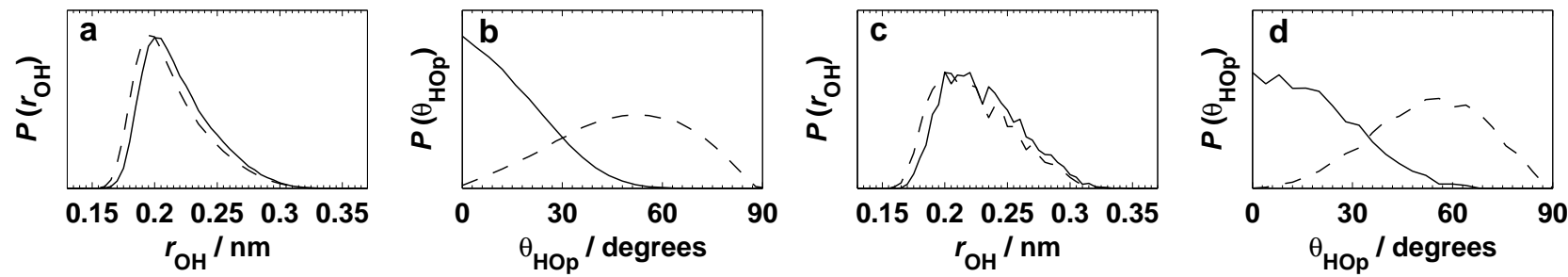

Figure 5: Probability distributions of TAs (a) distances $r_{\mathrm{OH}}$ of the planar (solid) and axial (dashed) hydrogens donating to its oxygen, and (b) the angles $\theta_{\mathrm{HOp}}$ of the respective $\mathrm{OH}$ vectors to the water's plane. (c) and (d) are the equivalent plots for QAs.

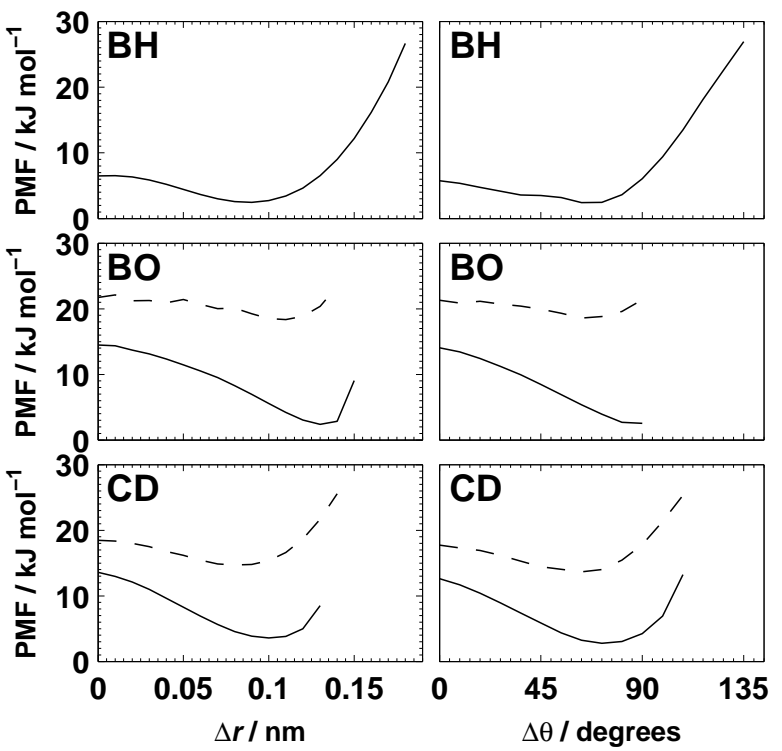

Figure 6: PMF profiles for the three transition states $\mathrm{BH}, \mathrm{BO}$ and $\mathrm{CD}$ as a function of the distance $\Delta r$ and angle $\Delta \theta$ reaction coordinates. The $\mathrm{BO}$ and $\mathrm{CD}$ plots are for donors with an $\mathrm{HB}$ (solid) and those with a broken HB (dashed). 

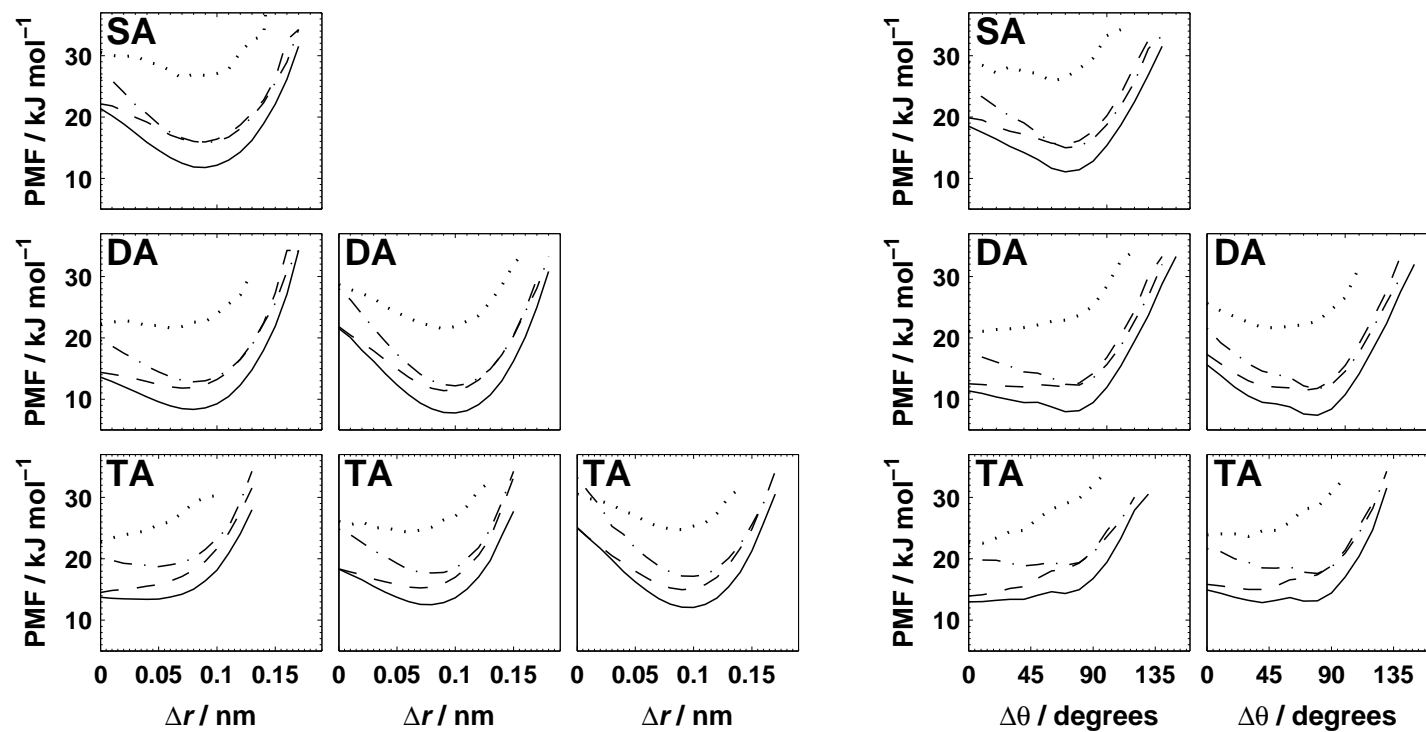

TA
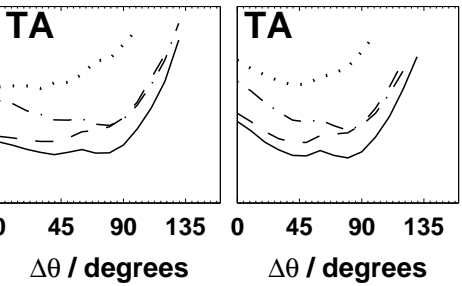

Figure 7: BH PMF profiles as a function of the distance $\Delta r$ and angle $\Delta \theta$ reaction coordinates for the donor to a SA, the outer and inner donors to a DA (left to right) and the outer, middle and inner donors to a TA (left to right). The next-nearest acceptor is either a NA (dotted), SA (dashed), DA (solid) or TA (dot-dashed).

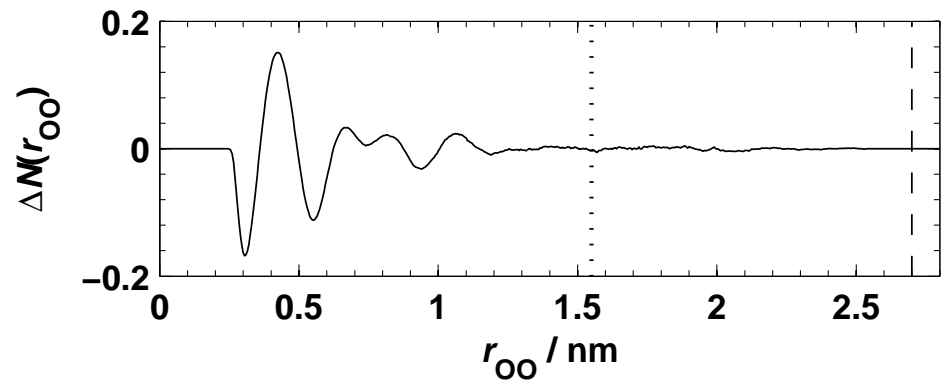

Figure 8: Cumulative donor-acceptor bias $\Delta N\left(r_{\mathrm{OO}}\right)$ versus $r_{\mathrm{OO}}$ averaged over all water molecules. The vertical lines are half the box edge (dotted) and half the box long diagonal (dashed). 